\title{
Intrusion Detection System Designed for Wireless using JADE Mobile Agent Framework
}

\author{
R. Sasikumar \\ Department of Computer Science and Engineering, \\ R.M.D Engineering College, Kavaraipettai. \\ Chennai, Tamil Nadu, India
}

\author{
D. Manjula \\ Department of Computer Science and Engineering, \\ College of Engineering, \\ Anna University \\ Chennai, Tamil Nadu, India
}

\begin{abstract}
In wireless environment the system allows the end users to use the application without installation and access their personal files at any computer with internet access. Apart from the advantages of wireless network, security is the major issue. Due to the distributed nature, wireless environment is an easy target for intruders looking for the possible attacks to exploit. To address the security issues in the wireless environment an Intrusion Detection System (IDS) is proposed based on the features of the mobile agent. The mobile agents are used to collect and analyze the data collected from wireless network to identify attacks exploited by the intruders.
\end{abstract}

\section{General Terms}

The main objective of the proposed system is to detect the known and unknown attacks exploited by the intruders in the wireless network.

\section{Keywords}

Keywords-Wireless computing, Intrusion Detection System, Mobile agent,intruders.

\section{INTRODUCTION}

Wireless computing means a type of parallel and distributed system consisting of a collection of interconnected computers that are dynamically provisioned and presented as one or more unified computing resources based on service level agreements established through negotiation between the service provider and consumer. In wireless environment the systems are distributed so there is greater chance of exploiting attacks by the intruders.

The intruders are the one who uses the services without any authorization and misuses the privileges. The intrusion detection means the process of detecting the individual who misuses the privileges assigned to them and one who access the data or service of legitimate user without any authorization. The intrusion detection system was designed to detect the intruders trying to exploit attacks in the network. In this proposed system the intrusion detection system uses the mobile agent to detect the attacks being exploited by the intruders.

An intrusion detection system (IDS) monitors network traffic and monitors for suspicious activity and alerts the system or network administrator. In some cases the IDS may also respond to anomalous or malicious traffic by taking action such as blocking the user or source IP address from accessing the network.

The goal of IDS is to detect suspicious traffic in different ways. There are network based (NIDS) [12] and host based (HIDS) intrusion detection systems. There are IDS that detect based on looking for specific signatures of known threatssimilar to the way antivirus software typically detects and protects against malware- and there are IDS that detect based on comparing traffic patterns against a baseline and looking for anomalies. There are IDS that simply monitor and alert and there are IDS that perform an action or actions in response to a detected threat.

An attack against a wireless computing system can be silent for network-based IDS [13] deployed in its environment, because node communication is usually encrypted. Attacks can also be invisible to host-based IDS, because specific attacks don't necessarily leave traces in nodes operating system, where the host -based IDS resides. In this way, traditional IDS can't appropriately identify suspicious attacks in wireless environment.

The mobile agent is an agent having the capability of moving from one host to another. It interacts with the other nodes to collect the data. The advantage of mobile agent technology are reduces the network overload, overcoming network latency, robust and fault tolerant and it works in heterogeneous environment. The mobile agent technology [11] has been shown to be very suitable to solve intrusion detection in a distributed environment. The proposed system employs the mobile agent technology to detect the known and unknown attacks exploited by the attacker.

\section{RELATED WORK}

The IDS should protect the system and needed to be able to resist attack and also needed to be fault tolerant, highly adaptable and configurable. According to the above characteristics, the agent technology is appropriate alternative to develop intrusion detection system. The mobile agent based intrusion detection system were developed [2] which uses the trace gray technique to detect the intrusions. A proposed efficient anomaly intrusion detection system in Ad-hoc by mobile agents[5] which uses the data mining algorithm to detect the attacks exploited by the intruders. Mobile agent based intrusion detection system for MANET [4] proposed by yinan $\mathrm{Li}$ which uses the clustering and joint detection technique to identify the intruders. Imen Brahmi proposed in a distributed mobile agent based intrusion detection system, called MAD-IDS[1], The architecture of the MAD-IDS is based on detection of known and unknown attacks which uses the clustering and rule mining technique. Intelligent intrusion detection system framework using mobile agents [3] which detects the intruders based on the user profile and process profile. Research on distributed intrusion detection system based on mobile agent [6], which increases the system flexibility and security. Signature based method [7] is used in distributed intrusion detection using mobile agents against DDoS attacks. S.S Sodhi proposed a distributed intrusion 
detection using aglet mobile agent technology [8] which uses the anomaly detection method. Trust modeling technique [9] is used in agent based network intrusion detection system which detects the intruders based on the trust established between the systems. Bin Dong proposed a intrusion detection system based on agents which uses the STAT technique [10] to detect the attacks.

\section{OVERVIEW OF IDS AND MOBILE AGENT}

\subsection{Intrusion detection system (IDS)}

Intrusion Detection [14] is the process of monitoring the events occurring in a computer system or network and analyzing them for signs of intrusions, like unauthorized entrance, activity, or file modification. There are three steps in the process of intrusion detection which are:

- Monitoring and analyzing traffic;

- Identifying abnormal activities;

- Assessing severity and raising alarm.

Intrusion Detection System (IDS) is software that automates the intrusion detection process and detects possible intrusions. Intrusion Detection Systems serve three essential security functions: they monitor, detect, and respond to unauthorized activity by company insiders and outsider intrusion. An IDS is composed of several components:

- Sensors which generate security events;

- Console to monitor events and alerts and control the sensors;

- Central Engine that records events logged by sensors in a database and uses a system of rules to generate alerts from security events received.

IDS tools aim to detect computer attacks and computer misuse, and to alert the proper individuals upon detection. IDSs use policies to define certain events that, if detected will issue an alert. Certain IDS have the capability of sending out alerts, so that the administrator of the IDS will receive a notification of a possible security incident in the form of a page, email, or SNMP trap. Many IDSs not only recognize a particular incident and issue an appropriate alert, they also respond automatically to the event. Such a response might include logging off a user, disabling a user account, and launching of scripts. IDS are an integral and necessary element of a complete information security infrastructure performing as "the logical complement to network firewalls".

\subsection{IDS Techniques}

There are four basic techniques used to detect intruders:

- Anomaly detection

- Misuse detection (signature detection)

- Target monitoring

- Stealth Probes

\subsection{Mobile Agent}

The software agent can be treated as Mobile agent [8], as they are able to migrate from one computer to another computer. The mobile agents are very powerful programs, which can act even in the absence of the machine that initiated them. After completion of their assigned tasks, the mobile agents return to the host machine to report the result or simply terminate. Useful Characteristic of Mobile agents are

1. Autonomy: Agents are independently running entities, they operate without human control.
2. Mobility: Agents are able to suspend processing on one platform and to move to another one where they resume execution.

3. Rationality: Agents embody the capacity to analyze and solve a problem in a rational manner.

4. Reactivity: Agents perceive their environment and adapt their behaviour in a dynamic way to match, as soon as possible, new environment parameters.

5. Inferential capability: Agents are able to share a set of knowledge in order to achieve a specific goal.

6. Pro-activeness: Agents can decide to adapt their behaviour to their environment,

7. Social ability: Agents are able to meet and interact with other agents. The interaction and collaboration between agents is achieved by an Agent Communication Language (ACL) and it may depend on ontology.

\subsection{Advantages of using Mobile Agents} listed below:

The advantages of using mobile agents in IDS are

- $\quad$ Minimizing the network traffic

- Structure and Platform independence

- Dynamic nature \& Scalable

- Operates in heterogeneous environment

- $\quad$ Robust \& Fault tolerant

- Overcomes network latency

\section{PROPOSED WORK}

\subsection{System Architecture}

The Intrusion detection system for this environment is proposed based on the mobile agent, which uses the data mining technique to detect the intrusions in the wireless environment. The following fig 1 contains various mobile agents for collecting and analyzing the data in the wireless environment. There are different agents used to detect the intrusions, they are as follows, Collector agent, Misuse detection agent, Anomaly detection agent, Classifier agent and alert agent.

These agents are used to collect and analyze the data collected from wireless environment to detect the attacks exploited by the intruders.

\subsection{System Description}

\subsubsection{Collector Agent}

The collector agent is the first agent to work in the system, since it connects to the network. It collects the data from the wireless environment and stores those data in the file. This file is given as an input to the misuse detection agent.

\subsubsection{Misuse Detection Agent}

The misuse detection agent is used to analyze the data captured by the collector agent. It detects the known attacks in network by using the pattern matching algorithm. If 


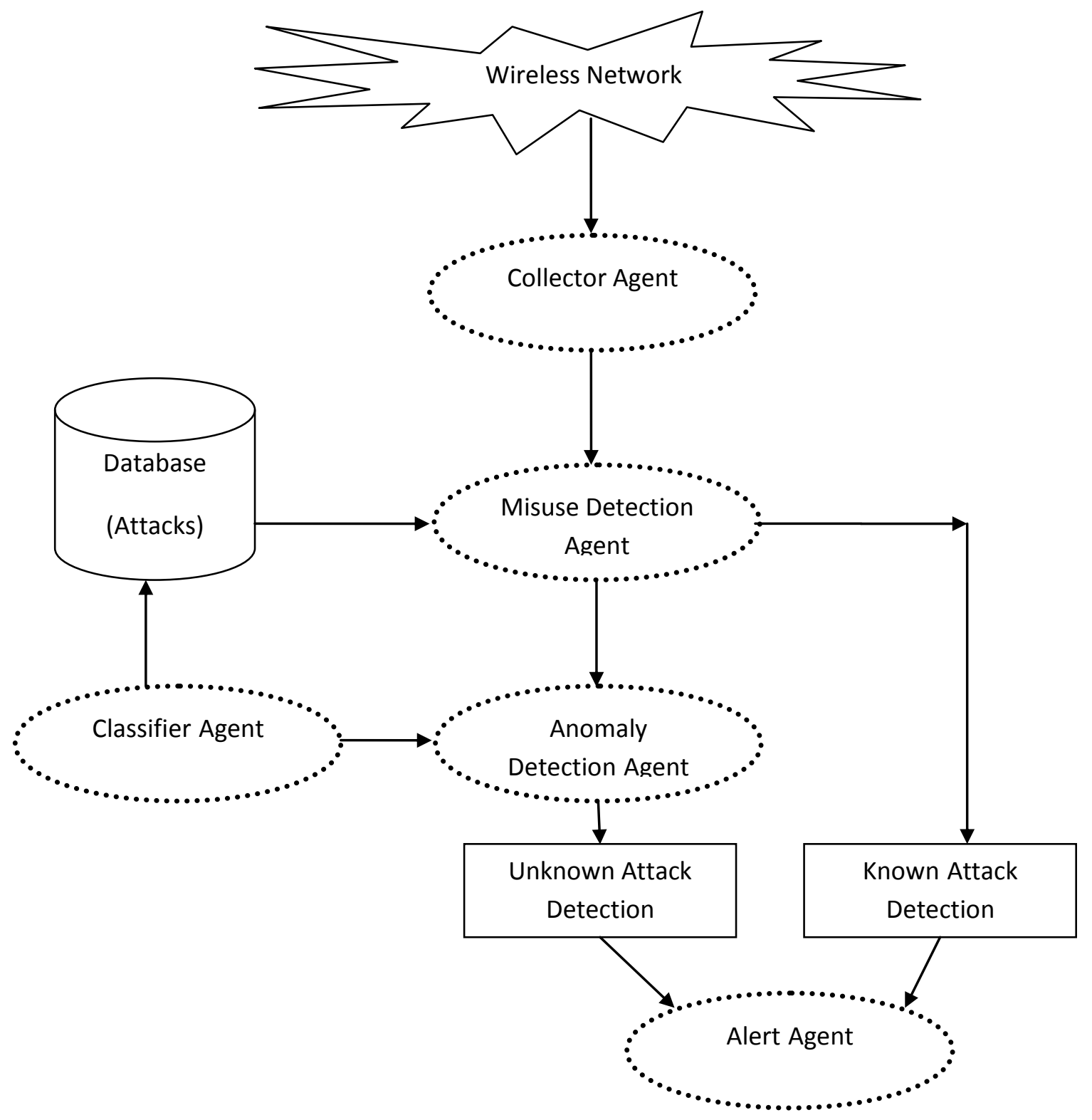

Fig 1: System Architecture

there is a similarity between the collected packets and attack signatures in the database, then it reports to alert agent.

\subsubsection{Anomaly Detection Agent}

The anomaly detection agent is used to detect the new or unknown attacks by using the classification techniques. The anomaly detection agent collects the data from the misuse detection agent to analyze the data to detect the unknown attacks, it feds the data to classifier agent to detect the new attack.

\subsubsection{Classifier Agent}

The classifier agent uses the naïve bayes classifier to detect the new attack. It classifies the data based on the dataset available in the database. If the incoming data is detected as attack means then it reports to anomaly agent, which in turn reports to alert agent about the attack. It updates the detected attack in the database.

\subsubsection{Alert Agent}

The alert agent is used to alert the system if any intrusion occurs in the network. It alerts the system based on the output of the misuse and anomaly detection agent.

\section{IMPLEMENTATION}

JADE (Java Agent Development framework) is a software framework which is used to implement the mobile agents for 
the proposed intrusion detection system. The collector agent is assigned a task to collect the data from the wireless environment and stores it as a file. The file is then forwarded to the misuse detection agent, it analyses the data by matching the collected data with the attacks available in the database and if the data is similar to the patterns in the database then it reports to alert agent. If the data is not matched with the database then the classifier agent is used to classify the data based on the dataset and then it reports to alert agent and updates the database about the attack. The alert agent alerts the system based on the outcome of misuse and anomaly detection agent.

\subsection{Algorithm}

Step 1: The threshold value is assigned by the user.

Step 2: The data received from the misuse detection agent is compared with the dataset available in database based on the threshold value.

Step 3: If the comparison of data exceeds the threshold value then it is detected as a attack and reports the anomaly detection agent.

Step 4: Then, the detected attack is updated in the database.

Step 5: The anomaly detection agent reports to the alert agent, then the alert is raised by the alert agent about the attack.

\subsection{Agent Platform}

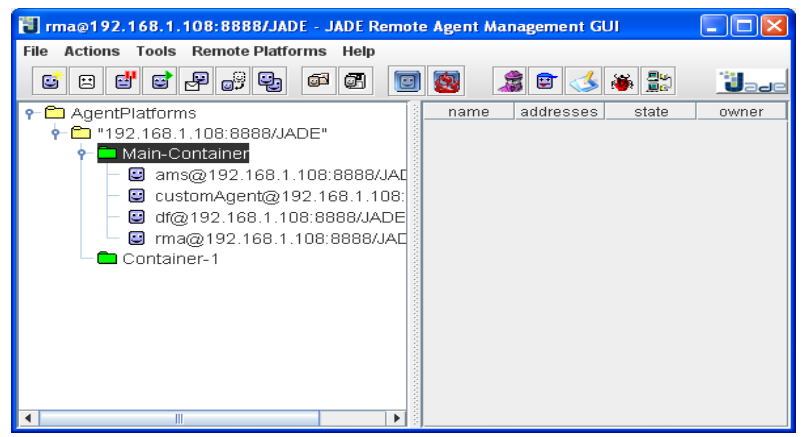

Fig 2: Agent platform

The above Fig 2 snapshot shows the JADE environment where the agents will be created and the task is assigned to the entire agent.

\subsection{To Add a Agent}

\begin{tabular}{l} 
NJ Insert Parameters \\
Agent Name \\
Container \\
\hline U are not sure about the implemementation of this agent, Cancel this operation. \\
\hline OK
\end{tabular}

Fig 3: Add a Agent

The above Fig 3 snapshot describes about the creation of the agents.

\subsection{After Creation of Agents}

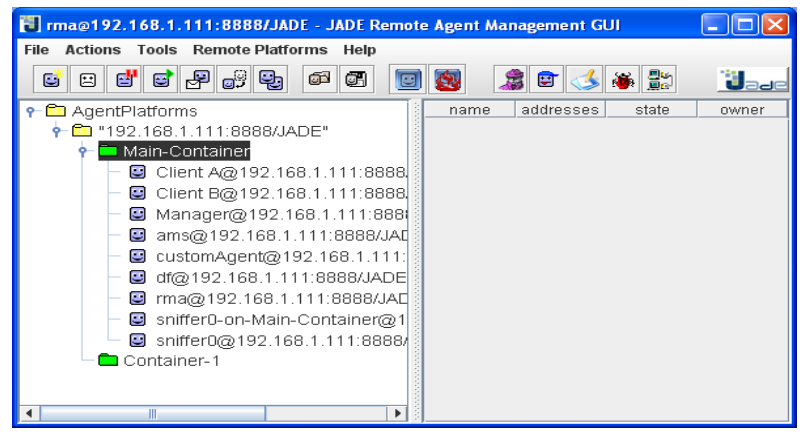

Fig 4: Agent platform after creation of Agents

The above Fig 4 snapshot shows the created agents with the name client $\mathrm{A}$ and client $\mathrm{B}$.

\subsection{Agent Communication}

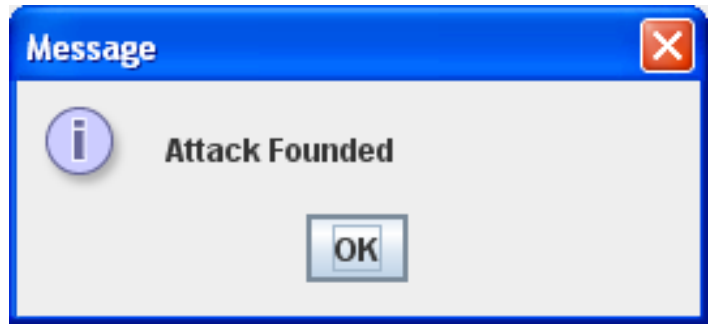

Fig 5: Agent platform after communication of Agents

The above Fig 5 snapshots show the communication between the clients of the local network, the client data are analysed to check for any attack by using misuse detection agent. If misuse detection agent finds any client data matches with attack defined in database then the alert agent alerts the system by sending a message.

\section{RESULT}

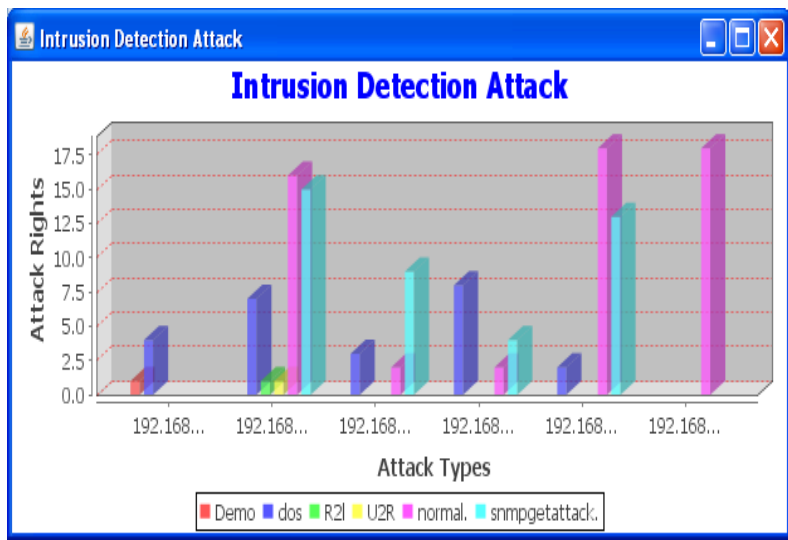

Fig 6: Intrusion Detection Attack

\section{ACKNOWLEDGMENTS}

The IDS for wireless computing is proposed which integrates the mobile agent to detect known and unknown attacks. All the mobile agents are configured in order to perform the operations like collecting the data from wireless environment, and these data are analyzed by the misuse detection agent. This agent checks whether the data collected are matching with the attack dataset available in the database. If any collected data is matched then the misuse detection agent informs the alert agent to alert the system about the intrusion. 
On the other hand, if the collected data is not matched with the dataset then the collected data are analyzed by anomaly detection agent, which uses naïve bayes classifier to detect the unknown or new attacks. The final result will be such that the known and unknown or new attacks are detected by the proposed architecture. Proper intrusion result techniques are required in order to protect the network system. How to work together the intrusion detection and response modules and how to take action to the predictable attacks effectively deserve further research. In this paper, simply used misusebased detection of a simulated Wireless network. A future possibility could be to detect ambiguous usage of a cloud environment network using anomaly detection techniques. In future plan to observe if the combination of classifiers and the creation of a group classifier can give us better results.

\section{REFERENCES}

[1] Imen Brahmi, Sadok Ben Yahia, and Pascal Poncelete, Vol 6122, pp:73-76, June 2010. "MAD-IDS: Novel Intrusion Detection System using Mobile Agents and Data Mining approaches" in Intelligence and Security Informatics, Pacific Asia Workshop, PAISI 2010.

[2] Ani Taggu, Amar Taggu, pp: 1-4, January 2011 "TraceGray: An Application layer scheme for intrusion detection in MANET using Mobile agents" in Third International Conference on Communication Systems and Networks.

[3] Esfandi. Vol 7, pp:73-77, July 2010. A, "Efficient anomaly intrusion detection system in Ad-hoc networks by Mobile Agents" in Third IEEE International Conference on Computer Science and Information Technology.

[4] Yinan Li, Zhihong Qian, pp: 145-148, March 2010. "Mobile agents based intrusion detection system for mobile Ad-hoc network" in International Conference on Innovative Computing and Communication.

[5] N.Jaisankar, R. Saravanan, K. Duraisamy, Vol 1, No 2, July 2009. "Intelligent intrusion detection system framework using mobile agents" in International Journal of Network Security and its Applications.
[6] Jin-Gang-Cao, Gu-Ping-Zheng, Vol 3, pp: 1394-1399, July 2008. "Research on distributed intrusion detection system based on mobile agents" in Seventh International Conference on Machine Learning and Cybermatics.

[7] Ugur Akyazi, pp: 1-6, October 2008. A. Sima Etaner Uyar, "Distributed Intrusion detection using mobile agents against DDoS attacks" in $23^{\text {rd }}$ International Symposium on Computer and Information Sciences.

[8] Manmeet Singh, S.S Sodhi, pp: 148-153, March 2007. "Distributed intrusion Detection using Aglet Mobile agent technology" in Proceedings of National Conference and Opportunities in Information Technology (COIT).

[9] Vojtech Krmicek, Pavel Celeda, Martin Rehak, Michael Pechoucek, pp: 528-531, August 2007. "Agent based network intrusion detection system" in International Conference on Intelligent Agent Technology.

[10] Bin-Dong, Xiu-Ling-Liu, Vol 6, August 2007. "An improved intrusion detection system based on agents" in Sixth International Conference on Machine Learning and Cybermatics.

[11] W. A. Jansen, Vol: 15, pp: 1392-1401, July 2002. "Intrusion Detection with Mobile Agents", in Computer communication,.

[12] Mell P, Karygiannis T, W. Jansen, pp: 1-12, June 2000. "Mobile Agents in Intrusion Detection and Response", in proceedings of the $12^{\text {th }}$ annual Canadian Information Technology Security Symposium.

[13] Kleber Vieira, Alexander Schulter, Carlos Becker Westphall, Carla Merkle Westphall, vol 2, Issue 4, pp:38-43, August 2010 ."Intrusion Detection for Grid and Wireless Computing" in IT professional sponsored by Computer Society of India.

[14] D.E. Denning, vol 13, Issue 2, pp:222-232, 1987. "An Intrusion Detection Model", in IEEE transactions on Software Engineering. 